PROCEEDINGS OF THE

AMERICAN MATHEMATICAL SOCIETY

Volume 128, Number 4, Pages 1087-1095

S 0002-9939(99)05251-X

Article electronically published on July 28, 1999

\title{
ASYMPTOTIC REGULARITY OF DAUBECHIES' SCALING FUNCTIONS
}

\author{
KA-SING LAU AND QIYU SUN \\ (Communicated by David R. Larson)
}

Abstract. Let $\phi_{N}, N \geq 1$, be Daubechies' scaling function with symbol $\left(\frac{1+e^{-i \xi}}{2}\right)^{N} Q_{N}(\xi)$, and let $s_{p}\left(\phi_{N}\right), 0<p \leq \infty$, be the corresponding $L^{p}$ Sobolev exponent. In this paper, we make a sharp estimation of $s_{p}\left(\phi_{N}\right)$, and we prove that there exists a constant $C$ independent of $N$ such that

$$
N-\frac{\ln \left|Q_{N}(2 \pi / 3)\right|}{\ln 2}-\frac{C}{N} \leq s_{p}\left(\phi_{N}\right) \leq N-\frac{\ln \left|Q_{N}(2 \pi / 3)\right|}{\ln 2} .
$$

This answers a question of Cohen and Daubeschies (Rev. Mat. Iberoamericana, 12(1996), 527-591) positively.

\section{INTRODUCTION}

For $N \geq 1$, let

$$
P_{N}(t)=\sum_{k=0}^{N-1}\left(\begin{array}{c}
N-1+k \\
k
\end{array}\right) t^{k} .
$$

Then

$$
(1-t)^{N} P_{N}(t)+t^{N} P_{N}(1-t)=1
$$

and $P_{N}$ is the unique polynomial solution of the equation with degree not greater than $N-1$.

Let $Q_{N}(\xi)$ be a trigonometric polynomial with real coefficients satisfying

$$
\left|Q_{N}(\xi)\right|^{2}=P_{N}\left(\sin ^{2} \frac{\xi}{2}\right) .
$$

It is known that such $Q_{N}$ exists by the Riesz Lemma, but $Q_{N}$ is not unique. Set

$$
H_{N}(\xi)=\left(\frac{1+e^{-i \xi}}{2}\right)^{N} Q_{N}(\xi)=\frac{1}{2} \sum_{k \in \mathbb{Z}} c_{k} e^{-i k \xi} .
$$

We are interested in the $Q_{N}$ such that the solution $\phi_{N}$ of the refinement equation

$$
\phi_{N}(x)=\sum_{k \in \mathbb{Z}} c_{k} \phi_{N}(2 x-k)
$$

Received by the editors November 3, 1997 and, in revised form, May 30, 1998.

1991 Mathematics Subject Classification. Primary 42C15, 26A15, 26A18, 39A10, 42A05.

Key words and phrases. Fourier transform, scaling function, Sobolev exponent, wavelet. 
with $\int_{\mathbb{R}} \phi_{N}(x) d x=1$ that generates an orthonormal basis of $L^{2}(\mathbb{R})$. The functions $\phi_{N}$ are the well known Daubechies' scaling functions [ [6]. For an integrable function $f$, we let $\hat{f}(\xi)=\int_{\mathbb{R}} f(x) e^{-i x \xi} d x$ be the Fourier transform of $f$. Then

$$
\widehat{\phi_{N}}(\xi)=H_{N}\left(\frac{\xi}{2}\right) \widehat{\phi_{N}}\left(\frac{\xi}{2}\right)
$$

and

$$
\widehat{\phi_{N}}(\xi)=\prod_{j=1}^{\infty} H_{N}\left(2^{-j} \xi\right)
$$

The regularity of the scaling functions has central importance in the theory of wavelets. In 14] Volkmer proved that the Hölder index of $\phi_{N}$ is $\left(1-\frac{\ln 3}{2 \ln 2}\right) N+o(N)$ as $N$ tends to infinity. Recently Bi, Dai and Sun ([1]) improved the estimation as

$$
\left(1-\frac{\ln 3}{2 \ln 2}\right) N+\frac{\ln N}{4 \ln 2}+O(1)
$$

Another popular approach to the regularity is to use the Sobolev exponent. Recall that the Sobolev exponent $s_{p}(f), 0<p<\infty$, is defined by

$$
s_{p}(f)=\sup \left\{s: \int_{\mathbb{R}}|\hat{f}(\xi)|^{p}(1+|\xi|)^{p s} d \xi<\infty\right\},
$$

and for $p=\infty$,

$$
s_{\infty}(f)=\sup \left\{s: \hat{f}(\xi)(1+|\xi|)^{s} \text { is a bounded function }\right\} .
$$

There is considerable literature devoted to estimating the Sobolev exponent for scaling functions in general, for example, 8] and [13] for $s_{2}(f)$, [2] for $s_{1}(f)$, [10] and 9 for $s_{p}(f)$ with $1 \leq p<\infty$, 12 for Triebel-Lizorkin space and Besov space, and [11] for $L^{p}$ Lipschitz space. For Daubechies' scaling functions, Volkmer [15] proved that

$$
N-\frac{\ln \left|Q_{N}(2 \pi / 3)\right|}{\ln 2}-\frac{1}{2} \leq s_{2}\left(\phi_{N}\right) \leq N-\frac{\ln \left|Q_{N}(2 \pi / 3)\right|}{\ln 2} .
$$

Recently, Cohen and Daubechies ([3], [7]) computed $s_{p}\left(\phi_{N}\right)$ for $p=1,2,4,8$ and $N=1,2, \cdots, 19$, and found that the difference of $s_{p}\left(\phi_{N}\right)$ between different $p$ becomes very small for $N$ large. Based on this observation, they asked

Problem. Let $\phi_{N}$ be defined by (2). For $0<p, q<\infty$, is it true that

$$
\lim _{N \rightarrow \infty}\left(s_{p}\left(\phi_{N}\right)-s_{q}\left(\phi_{N}\right)\right)=0 ?
$$

In this paper, we answer this question affirmatively and generalize the estimation in 15 in part.

Theorem. Let $\phi_{N}$ be defined by (2). For $0<p<\infty$, there exists a constant $C$ independent of $N$ such that

$$
N-\frac{\ln \left|Q_{N}(2 \pi / 3)\right|}{\ln 2}-\frac{C}{N} \leq s_{p}\left(\phi_{N}\right) \leq N-\frac{\ln \left|Q_{N}(2 \pi / 3)\right|}{\ln 2},
$$

and for $p=\infty$,

$$
s_{\infty}\left(\phi_{N}\right)=N-\frac{\ln \left|Q_{N}(2 \pi / 3)\right|}{\ln 2} .
$$




\begin{tabular}{|c|l|l|l|l|}
\hline$N$ & $p=1$ & $p=2$ & $p=8$ & $N-\frac{\ln \left|Q_{N}(2 \pi / 3)\right|}{\ln 2}$ \\
\hline 2 & 0.521293 & 0.999820 & 1.310014 & 1.339036 \\
\hline 3 & 0.979675 & 1.414947 & 1.631688 & 1.636040 \\
\hline 4 & 1.391644 & 1.775305 & 1.912144 & 1.912537 \\
\hline 5 & 1.767934 & 2.096541 & 2.174682 & 2.176608 \\
\hline 6 & 2.116733 & 2.388060 & 2.431755 & 2.432246 \\
\hline 7 & 2.441544 & 2.658569 & 2.680307 & 2.681743 \\
\hline 8 & 2.746639 & 2.914556 & 2.925926 & 2.926549 \\
\hline 9 & 3.035292 & 3.161380 & 3.165533 & 3.167644 \\
\hline 10 & 3.309107 & 3.402546 & 3.405141 & 3.405724 \\
\hline 11 & 3.572141 & 3.639569 & 3.638529 & 3.641301 \\
\hline 12 & 3.825525 & 3.873991 & 3.871917 & 3.874766 \\
\hline 13 & 4.071021 & 4.105802 & 4.105305 & 4.106422 \\
\hline 14 & 4.311641 & 4.336042 & 4.335502 & 4.336511 \\
\hline 15 & 4.547368 & 4.564708 & 4.562449 & 4.565229 \\
\hline 16 & 4.780028 & 4.792323 & 4.792645 & 4.792735 \\
\hline 17 & 5.010231 & 5.018884 & 5.016283 & 5.019164 \\
\hline 18 & 5.238588 & 5.244390 & 5.243230 & 5.244627 \\
\hline 19 & 5.464480 & 5.468841 & 5.466868 & 5.469221 \\
\hline
\end{tabular}

In the table, we list the approximate value of the $L^{p}$ Sobolev exponent $s_{p}\left(\phi_{N}\right)$. The first three columns $s_{p}\left(\phi_{N}\right), p=1,2,8$, are obtained by Cohen and Daubechies in [3]. The last column $N-\frac{\ln \left|Q_{N}(2 \pi / 3)\right|}{\ln 2}$ is the approximate value from the theorem. Note that the numerical data matches with the theorem.

\section{UPPER BOUND ESTIMATION}

In this section, we will prove the upper bound estimate of $s_{p}\left(\phi_{N}\right)$.

Proposition 1. Let $\phi_{N}$ be defined by (2). Then for $0<p \leq \infty$,

$$
s_{p}\left(\phi_{N}\right) \leq N-\frac{\ln \left|Q_{N}(2 \pi / 3)\right|}{\ln 2} .
$$

Proof. It follows from (3) that

$$
\left|\widehat{\phi_{N}}\left(\frac{2^{k} \pi}{3}\right)\right|=2^{-(k-1) N}\left|Q_{N}\left(\frac{2 \pi}{3}\right)\right|^{k-1}\left|\widehat{\phi_{N}}\left(\frac{2 \pi}{3}\right)\right| .
$$

Hence (5) holds for $p=\infty$.

To prove the case for $0<p<\infty$, we let $\tilde{\phi}_{N}$ be the compactly supported distribution defined by

$$
\widehat{\widetilde{\phi}_{N}}(\xi)=\prod_{j=1}^{\infty} Q_{N}\left(\xi / 2^{j}\right) .
$$

Let $n_{k}=\left(4^{k}-1\right) / 3$; then by a similar method as used in Proposition 3 in [4, we obtain for any $\epsilon>0$ there exists a constant $C$ such that for $\xi \in[-\pi, \pi]$ and for sufficiently large $k$,

$$
\left|\widehat{\tilde{\phi}_{N}}\left(\xi+2 n_{k} \pi\right)\right| \geq C\left|Q_{N}\left(\frac{2 \pi}{3}\right)\right|^{2 k} 4^{-k \epsilon} .
$$


Since

$$
\widehat{\phi_{N}}(\xi)=\prod_{j=1}^{\infty}\left(\frac{1+e^{-i 2^{-j} \xi}}{2}\right)^{N}{\widetilde{\phi_{N}}}_{(\xi)}\left(\frac{1-e^{-i \xi}}{i \xi}\right)^{N}{\widetilde{\phi_{N}}}_{(\xi)}
$$

there exists an integer $k_{0}$ such that for $\xi \in\left[\frac{5 \pi}{9}, \frac{7 \pi}{9}\right]$ and $k \geq k_{0}$,

$$
\left|\widehat{\phi_{N}}\left(\xi+2 n_{k} \pi\right)\right| \geq C 4^{-N k-\epsilon k}\left|Q_{N}\left(\frac{2 \pi}{3}\right)\right|^{2 k} .
$$

Obviously

$$
\int_{\mathbb{R}}\left|\widehat{\phi_{N}}(\xi)\right|^{p}(1+|\xi|)^{p s} d \xi<\infty
$$

implies that

$$
\int_{\left[\frac{5 \pi}{9}, \frac{7 \pi}{9}\right]+2 n_{k} \pi}\left|\widehat{\phi_{N}}(\xi)\right|^{p}(1+|\xi|)^{p s} d \xi
$$

is bounded on $k$. Hence there exists a constant $C$ such that $4^{(s-N-\epsilon) k p}\left|Q_{N}\left(\frac{2 \pi}{3}\right)\right|^{2 k p}$ $\leq C$ for all $k$. This implies that

$$
s-N-\frac{\ln \left|Q_{N}(2 \pi / 3)\right|}{\ln 2}-\epsilon \leq 0
$$

and (5) follows from the definition of $s_{p}\left(\phi_{N}\right), 0<p<\infty$.

\section{LOWER BOUND ESTIMATION}

In this section, we prove the lower bound estimate for $s_{p}\left(\phi_{N}\right)$.

Proposition 2. Let $\phi_{N}$ be defined by (2). Then for $0<p<\infty$ and for any integer $M \geq 2$ there exist a constant $1 / 2<r<1$ and an integer $N_{0}$ independent of $p$ and $M$ such that for $N \geq N_{0}$,

$$
s_{p}\left(\phi_{N}\right) \geq N-\frac{p M \ln \left|Q_{N}(2 \pi / 3)\right|+\ln \left(2+2^{M} r^{N p}\right)}{p M \ln 2} .
$$

Also

$$
s_{\infty}\left(\phi_{N}\right) \geq N-\frac{\ln \left|Q_{N}(2 \pi / 3)\right|}{\ln 2} .
$$

Obviously our main theorem follows from Propositions 1 and 2 by choosing the above $M$ as the integral part of $-p N \ln r / \ln 2$. We need some lemmas to prove the proposition. The main estimate is Lemma 6 , based on the accurate estimates of $Q_{N}(\xi)$ on $\left[0, \frac{2 \pi}{3}\right)$ and $Q_{N}(\xi) Q_{N}(2 \xi)$ on $\left[\frac{2 \pi}{3}, \pi\right]$. First we introduce an auxiliary function

$$
g(\xi)= \begin{cases}\left(\cos \frac{\xi}{2}\right)^{-2}, & |\xi| \leq \frac{\pi}{2}, \\ 4\left(\sin \frac{\xi}{2}\right)^{2}, & \frac{\pi}{2} \leq|\xi| \leq \pi, \\ g(\xi-2 m \pi), & \xi \in 2 m \pi+[-\pi, \pi] .\end{cases}
$$

Lemma 3. There exists a constant $C$ independent of $N$ and $\xi$ such that

$$
C^{-1} N^{-C} g(\xi)^{N} \leq\left|Q_{N}(\xi)\right|^{2} \leq g(\xi)^{N} .
$$

Proof. The right inequality was proved by Cohen and Séré [5, Lemma 2.3]. It remains to prove the left inequality. Write

$$
a_{k}(\xi)=\left(\begin{array}{c}
N-1+k \\
k
\end{array}\right)\left(\sin \frac{\xi}{2}\right)^{2 k}, \quad 0 \leq k \leq N-1 .
$$


Then

$$
\frac{a_{k}(\xi)}{a_{k-1}(\xi)}=\frac{N+k-1}{k} \sin ^{2} \frac{\xi}{2}
$$

Let $k_{0}$ be the integral part of $(N-1) \tan ^{2} \frac{\xi}{2}$. Then by observing that

$$
\frac{a_{k}(\xi)}{a_{k-1}(\xi)} \geq 1 \quad \text { if and only if } \quad k \leq(N-1) \tan ^{2} \frac{\xi}{2}
$$

and that $\left|\tan \frac{\xi}{2}\right| \leq 1$ for $|\xi| \leq \frac{\pi}{2}$, we have

$$
\max _{1 \leq k \leq N-1} a_{k}(\xi)=a_{k_{0}}(\xi), \quad|\xi| \leq \pi / 2 .
$$

By using the Stirling formula

$$
k !=k^{k} e^{-k} \sqrt{2 \pi k}(1+o(1))
$$

we have for $|\xi| \leq \pi / 2$,

$$
a_{k_{0}}(\xi)=\frac{\left(N+k_{0}-1\right) !}{k_{0} !(N-1) !}\left(\sin \frac{\xi}{2}\right)^{2 k_{0}}=\frac{\left(N+k_{0}-1\right)^{N+k_{0}-1}}{k_{0}^{k_{0}}(N-1)^{N-1}}\left(\sin \frac{\xi}{2}\right)^{2 k_{0}} B_{N}
$$

where $C^{-1} N^{-C} \leq B_{N} \leq C N^{C}$. By substituting $-1<k_{0}-(N-1) \tan ^{2} \frac{\xi}{2} \leq 0$ into the above expression and simplifying, we have

$$
a_{k_{0}}(\xi)=\tilde{B}_{N}\left(\cos \frac{\xi}{2}\right)^{-2 N}=\tilde{B}_{N} g(\xi)^{N}, \quad|\xi| \leq \pi / 2
$$

where $\left(C^{\prime}\right)^{-1} N^{-C^{\prime}} \leq \tilde{B}_{N} \leq C^{\prime} N^{C^{\prime}}$. This yields the left inequality of (7) for $|\xi| \leq \pi / 2$.

For $\frac{\pi}{2} \leq|\xi| \leq \pi, \tan ^{2} \frac{\xi}{2} \geq 1$ implies that

$$
a_{0}(\xi) \leq a_{1}(\xi) \leq \cdots \leq a_{N-1}(\xi) .
$$

By using the Stirling formula again and making a similar estimation, we have

$$
C^{-1} N^{-C} g(\xi)^{N}=C^{-1} N^{-C}\left(2 \sin \frac{\xi}{2}\right)^{2 N} \leq a_{N-1}(\xi) \leq\left|Q_{N}(\xi)\right|^{2}, \quad \frac{\pi}{2} \leq|\xi| \leq \pi,
$$

which completes the proof.

Lemma 4. Let $g(\xi)$ be defined by (6). Then

$$
0 \leq g(\xi) g(2 \xi) \leq\left|g\left(\frac{2 \pi}{3}\right)\right|^{2}, \quad|\xi| \in\left[\frac{2 \pi}{3}, \pi\right],
$$

and for $0<\delta<\frac{\pi}{6}$ there exists $0<r_{1}<1$ such that

$$
0 \leq g(\xi) g(2 \xi) \leq r_{1}^{2}\left|g\left(\frac{2 \pi}{3}\right)\right|^{2}, \quad|\xi| \in\left[\frac{2 \pi}{3}+\delta, \pi\right] .
$$

Proof. Recall that $g(\xi)$ is an even periodic function, hence it suffices to prove (8) for $\xi \in[0, \pi]$. Note that

$$
g(\xi) g(2 \xi)= \begin{cases}16 \sin ^{2} \frac{\xi}{2} \sin ^{2} \xi, & \xi \in\left[\frac{2 \pi}{3}, \frac{3 \pi}{4}\right], \\ 4 \sin ^{2} \frac{\xi}{2} \cos ^{-2} \xi, & \xi \in\left[\frac{3 \pi}{4}, \pi\right] .\end{cases}
$$

It is easy to check that the product is strictly decreasing on $\left[\frac{2 \pi}{3}, \pi\right]$. Hence

$$
0 \leq g(\xi) g(2 \xi) \leq g\left(\frac{2 \pi}{3}\right) g\left(\frac{4 \pi}{3}\right)=\left|g\left(\frac{2 \pi}{3}\right)\right|^{2} .
$$

The second part follows from the strictly decreasing property. 

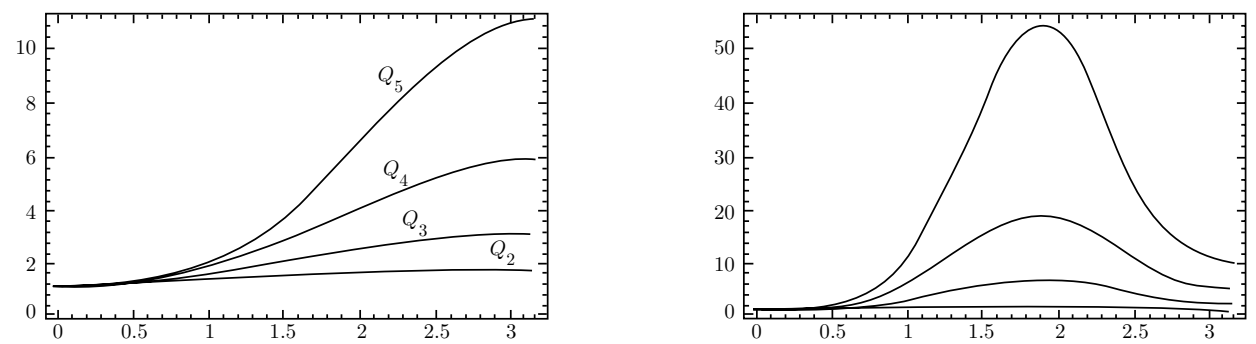

Figure 1.

Lemma 5. For any integer $N \geq 1$,

$$
\begin{aligned}
\left|Q_{N}(\xi)\right| & \leq\left|Q_{N}\left(\frac{2 \pi}{3}\right)\right|, \quad|\xi| \in\left[0, \frac{2 \pi}{3}\right), \\
\left|Q_{N}(\xi) Q_{N}(2 \xi)\right| & \leq\left|Q_{N}\left(\frac{2 \pi}{3}\right)\right|^{2}, \quad|\xi| \in\left[\frac{2 \pi}{3}, \pi\right) .
\end{aligned}
$$

Furthermore for any $0<\delta<\pi / 6$, there exists $0<r_{2}<1$ and an integer $N_{1}$ such that for $N>N_{1}$,

$$
\begin{aligned}
\left|Q_{N}(\xi)\right| & \leq r_{2}^{N}\left|Q_{N}\left(\frac{2 \pi}{3}\right)\right|, \quad|\xi| \in\left[0, \frac{2 \pi}{3}-\delta\right), \\
\left|Q_{N}(\xi) Q_{N}(2 \xi)\right| & \leq r_{2}^{N}\left|Q_{N}(2 \pi / 3)\right|^{2}, \quad|\xi| \in\left[\frac{2 \pi}{3}+\delta, \pi\right] .
\end{aligned}
$$

Proof. The first two inequalities were proved in [6. p. 222]. We use Lemma 3 to prove (12): for $|\xi| \in\left[0, \frac{2 \pi}{3}-\delta\right]$, there exists $0<r<1$ such that

$$
\left|Q_{N}(\xi)\right|^{2} \leq g(\xi)^{N} \leq r^{N} g\left(\frac{2 \pi}{3}\right)^{N} \leq C N^{C} r^{N}\left|Q_{N}\left(\frac{2 \pi}{3}\right)\right|^{2} .
$$

We pick $r_{2}$ so that $0<r<r_{2}<1$. Hence (12) holds for $N$ large enough. The proof of (13) is similar by using Lemma 4.

In regard to the above lemma, we include the graphs of $Q_{N}(\xi)$ and $Q_{N}(\xi) Q_{N}(2 \xi)$, $N=2,3,4,5$, for the convenience of the reader (see Figure 1).

For any $0<\delta<\pi / 6$ and $\xi \in R$, we define

$$
I_{k}(\xi, \delta)=\left\{j: 1 \leq j \leq k, 2^{j} \xi \in \bigcup_{m \in \mathbb{Z}}\left[-\frac{2 \pi}{3}+\delta, \frac{2 \pi}{3}-\delta\right]+2 m \pi\right\}
$$

and let $i_{k}(\xi, \delta)$ be the number of elements of $I_{k}(\xi, \delta)$.

Lemma 6. Let $N_{1}$ be as in Lemma 5. Then there exists a constant $C_{N}$ and a constant $0<r_{3}<1$ depending on $0<\delta<\pi / 6$ only, such that for $k>2$ and $N \geq N_{1}$

$$
\prod_{j=1}^{k}\left|Q_{N}\left(2^{j} \xi\right)\right| \leq C_{N} r_{3}^{N i_{k}(\xi, \delta)}\left|Q_{N}\left(\frac{2 \pi}{3}\right)\right|^{k} .
$$

Proof. We use $r_{2}(\delta)$ to denote the $r_{2}$ in Lemma 5, and choose $r_{3}(\delta)$ so that $r_{2}(\delta)$, $r_{2}(\delta / 2)<r_{3}(\delta)<1$. It is easy to see that by letting $C_{N}$ be large enough, the lemma holds for $k=1$ and $k=2$. We assume that (14) holds for $k<l$ with $l \geq 3$. For $k=l$, we divide the proof into four cases: 
(i) If $2 \xi \in\left[-\frac{2 \pi}{3}+\delta, \frac{2 \pi}{3}-\delta\right]+2 m \pi$, then $i_{k}(\xi, \delta)=i_{k-1}(2 \xi, \delta)+1$. We can write

$$
\prod_{j=1}^{k}\left|Q_{N}\left(2^{j} \xi\right)\right|=\left|Q_{N}(2 \xi)\right| \prod_{j=1}^{k-1}\left|Q_{N}\left(2^{j}(2 \xi)\right)\right|,
$$

and (14) follows from (12) with $r_{2}(\delta)<r_{3}(\delta)<1$ and the induction hypothesis.

(ii) If $2 \xi \in\left(\left[-\frac{2 \pi}{3},-\frac{2 \pi}{3}+\delta\right) \cup\left(\frac{2 \pi}{3}-\delta, \frac{2 \pi}{3}\right]\right)+2 m \pi$, then $i_{k}(\xi, \delta)=i_{k-1}(2 \xi, \delta)$ and the same induction hypothesis together with (10) implies (14).

(iii) If $2 \xi \in\left(\left[-\frac{2 \pi}{3}-\frac{\delta}{2},-\frac{2 \pi}{3}\right) \cup\left(\frac{2 \pi}{3}, \frac{2 \pi}{3}+\frac{\delta}{2}\right]\right)+2 m \pi$, then it follows that $2 \xi, 4 \xi \notin \bigcup_{m \in \mathbb{Z}}\left[-\frac{2 \pi}{3}+\delta, \frac{2 \pi}{3}-\delta\right]+2 m \pi$, hence $i_{k}(\xi, \delta)=i_{k-2}(4 \xi, \delta)$. Write

$$
\prod_{j=1}^{k}\left|Q_{N}\left(2^{j} \xi\right)\right|=\left|Q_{N}(2 \xi) Q_{N}(4 \xi)\right| \prod_{j=1}^{k-2}\left|Q_{N}\left(2^{j}(4 \xi)\right)\right|
$$

and (14) follows from (11).

(iv) If $2 \xi \in\left(\left[-\pi,-\frac{2 \pi}{3}-\frac{\delta}{2}\right) \cup\left(\frac{2 \pi}{3}+\frac{\delta}{2}, \pi\right]\right)+2 m \pi$, then $i_{k}(\xi, \delta) \leq i_{k-2}(4 \xi, \delta)+1$. By using the above product, $r_{2}(\delta / 2)<r_{3}(\delta)<1$ and (13), we have

$$
\prod_{j=1}^{k}\left|Q_{N}\left(2^{j} \xi\right)\right| \leq r_{2}\left(\frac{\delta}{2}\right)^{N} C_{N} r_{3}^{N i_{k-2}(4 \xi, \delta)}\left|Q_{N}\left(\frac{2 \pi}{3}\right)\right|^{k} \leq C_{N} r_{3}^{N i_{k}(\xi, \delta)}\left|Q_{N}\left(\frac{2 \pi}{3}\right)\right|^{k} .
$$

The induction step follows from these four cases.

For any integer $M \geq 2, k \geq 1$ and $\epsilon=\left(\epsilon_{1}, \epsilon_{2}, \cdots, \epsilon_{k M}\right)$ with $\epsilon_{i}=0$ or 1 , let $\alpha_{k M}(\epsilon)$ be the cardinality of the set

$$
A_{k M}(\epsilon)=\left\{l: 1 \leq l \leq k,\left(\epsilon_{(l-1) M+1}, \cdots, \epsilon_{l M}\right) \text { has two consecutive } 0 \text { or } 1\right\} .
$$

Then $\alpha_{k M}(\epsilon)=\sum_{l=0}^{k-1} \alpha_{M}\left(\epsilon^{l}\right)$ where $\epsilon^{l}=\left(\epsilon_{l M+1}, \cdots, \epsilon_{(l+1) M}\right)$ and

$$
\begin{gathered}
\sum_{\epsilon=\left(\epsilon_{1}, \cdots, \epsilon_{k M}\right) \in\{0,1\}^{k M}} r^{\alpha_{k M}(\epsilon)}=\sum_{l=0}^{k-1} \sum_{\epsilon^{l}=\left(\epsilon_{M l+1}, \cdots, \epsilon_{M(l+1)}\right) \in\{0,1\}^{M}} \prod_{j=0}^{k-1} r^{\alpha_{M}\left(\epsilon^{j}\right)} \\
=\left(\sum_{\epsilon=\left(\epsilon_{1}, \cdots, \epsilon_{M}\right) \in\{0,1\}^{M}} r^{\alpha_{M}(\epsilon)}\right)^{k}=\left(2+\left(2^{M}-2\right) r\right)^{k},
\end{gathered}
$$

where $r>0$ and the last equality follows from the fact that $\alpha_{M}(\epsilon)=1$ for any $\epsilon \in\{0,1\}^{M}$ except $\epsilon=(0,1,0,1, \cdots) \in\{0,1\}^{M}$ or $(1,0,1,0, \cdots) \in\{0,1\}^{M}$.

Lemma 7. Let $0<\delta<\pi / 6$. For $\xi \in[\pi, 2 \pi)$, write $\xi=2 \pi\left(\sum_{j=1}^{k M} \epsilon_{j} 2^{-j}+\eta\right)$ with $0 \leq \eta<2^{-k M}$. Then

$$
\alpha_{k M}(\epsilon)-1 \leq i_{k M}(\xi, \delta)
$$

Proof. Suppose $l \in A_{k M}(\epsilon)$ and $l \geq 2$. Then there exists an index $j \geq 2$ such that $(l-1) M+1 \leq j \leq l M-1$ and $\epsilon_{j}=\epsilon_{j+1}$. Hence

$$
2^{j-1} \xi=2 m \pi+2 \pi\left(\frac{\epsilon_{j}}{2}+\frac{\epsilon_{j+1}}{4}+\eta^{\prime}\right)
$$

for some integer $m$ and $0 \leq \eta^{\prime}<1 / 4$. For $\epsilon_{j}=\epsilon_{j+1}=0$,

$$
2 \pi\left(\frac{\epsilon_{j}}{2}+\frac{\epsilon_{j+1}}{4}+\eta^{\prime}\right) \in\left[-\frac{\pi}{2}, \frac{\pi}{2}\right]
$$


and for $\epsilon_{j}=\epsilon_{j+1}=1$,

$$
2 \pi\left(\frac{\epsilon_{j}}{2}+\frac{\epsilon_{j+1}}{4}+\eta^{\prime}-1\right) \in\left[-\frac{\pi}{2}, \frac{\pi}{2}\right] .
$$

Hence $2^{j-1} \xi \in \bigcup_{m \in \mathbb{Z}}[-2 \pi / 3+\delta, 2 \pi / 3-\delta]+2 m \pi$, i.e., $j-1 \in I_{k M}(\xi, \delta)$. What we have just shown is that each $l \in A_{k M}(\epsilon)$ corresponds to at least one distinct $j \in I_{k M}(\xi, \delta)$ provided that $l \geq 2$. The lemma follows from this assertion.

Proof of Proposition 2. Recall that

$$
\hat{\phi}_{N}(\xi)=\left(\frac{1-e^{-i \xi}}{i \xi}\right)^{N} \prod_{j=1}^{\infty} Q_{N}\left(\xi / 2^{j}\right) .
$$

Let $r=r_{3}(\pi / 6)$. Then for $\xi \in\left[2^{k M-1} \pi, 2^{k M} \pi\right]$ and $N \geq N_{1}$, Lemma 6 implies that

$$
\begin{aligned}
\left|\hat{\phi}_{N}(\xi)\right| & \leq C 2^{-k M N} \prod_{j=1}^{k M-1}\left|Q_{N}\left(2^{j-k M} \xi\right)\right| \\
& \leq C^{\prime} 2^{-k M N} r^{N i_{k M}\left(2^{-k M} \xi, \pi / 6\right)}\left|Q_{N}\left(\frac{2 \pi}{3}\right)\right|^{k M},
\end{aligned}
$$

where $C^{\prime}$ depends on $N$ only. It now follows from (3), (16) and (15) that

$$
\begin{aligned}
& \int_{2^{k M-1} \pi}^{2^{(k+1) M-1} \pi}\left|\hat{\phi}_{N}(\xi)\right|^{p} d \xi=\sum_{l=0}^{M-1} \int_{2^{k M-1+l} \pi}^{2^{k M+l} \pi}\left|\hat{\phi}_{N}(\xi)\right|^{p} d \xi \leq 2^{M} \int_{2^{k M-1} \pi}^{2^{k M} \pi}\left|\hat{\phi}_{N}(\xi)\right|^{p} d \xi \\
& \leq C^{\prime} 2^{-N k M p}\left|Q_{N}\left(\frac{2 \pi}{3}\right)\right|^{k M p} \int_{2^{k M-1} \pi}^{2^{k M} \pi} r^{N p i_{k M}\left(2^{-k M} \xi, \pi / 6\right)} d \xi \\
& \leq C^{\prime \prime} 2^{-N k M p}\left|Q_{N}\left(\frac{2 \pi}{3}\right)\right|^{k M p} \sum_{\epsilon_{j} \in\{0,1\}, 1 \leq j \leq k M} r^{N p \alpha_{k M}(\epsilon)} \\
& \leq C^{\prime \prime} 2^{-N k M p}\left|Q_{N}\left(\frac{2 \pi}{3}\right)\right|^{k M p}\left(2+2^{M} r^{N p}\right)^{k} .
\end{aligned}
$$

This completes the proof.

\section{ACKNOWLEDGEMENT}

The authors would like to thank the anonymous referee for his (her) suggestion. The research is supported by the Institute of Mathematical Sciences, CUHK and HKRGC earmark grant. The second author is also partially supported by the National Natural Sciences Foundation of China \# 69735020, the Tian Yuan Project of the National Natural Sciences Foundation of China \# 19631080, the Doctoral Bases Promotion Foundation of National Educational Commission of China \# 97033519 and the Zhejiang Provincial Sciences Foundation of China \# 196083.

\section{REFERENCES}

[1] N. Bi, X. Dai and Q. Sun, Construction of compactly supported $M$-band wavelets, Appl. Comp. Harmonic Anal., to appear.

[2] A. Cohen and I. Daubechies, Non-separable bidimensional wavelet bases, Rev. Mat. Iberoamericana, 9(1983), 51-137. MR 94k:42047

[3] A. Cohen and I. Daubechies, A new method to determine the regularity of refinable functions, Rev. Mat. Iberoamericana, 12(1996), 527-591.

[4] A. Cohen, I. Daubechies and A. Ron, How smooth is the smoothness function in a given refinable space?, Appl. Comput. Harmonic Anal., 3(1996), 87-89. 
[5] A. Cohen and E. Séré, Time-frequency localization with non-stationary wavelet packet, Preprint.

[6] I. Daubechies, Ten Lectures on Wavelets, CBMS-Conference Lecture Notes, No. 61, SIAM Philadelphia, 1992. MR 93e:42045

[7] I. Daubechies, Using Fredholm determinants to estimate the smoothness of refinable functions, In Approximation Theory VIII, Vol.2, Wavelet and Multilevel Approximation, Edited by C. K. Chui and L. L. Schumaker, World Scientific Press, 1995, pp. 89-122. MR 98e:42032

[8] T. Eirola, Sobolev characterization of solutions of dilation equation, SIAM J. Math. Anal., 23(1992), 1015-1030. MR 93f:42056

[9] A. Fan and K. S. Lau, Asymptotic behavior of multiperiodic periodic functions $G(x)=$ $\prod_{n=1}^{\infty} g\left(x / 2^{n}\right)$, J. Four. Anal. and Appl., 4(1998), 130-150.

[10] L. Hervé, Construction et régularité des fonctions d'échelle, SIAM J. Math. Anal., 26(1995), 1361-1385. MR 97j:42015

[11] K. S. Lau and J. Wang, Characterization of $L^{p}$-solution for the two-scale dilation equations, SIAM J. Math. Anal., 26(1995), 1018-1046. MR 96f:39004

[12] B. Ma and Q. Sun, Compactly supported refinable distribution in Triebel-Lizorkin space and Besov space, J. Fourier Anal. Appl., to appear.

[13] L. F. Villemoes, Wavelet analysis of refinement equations, SIAM J. Math. Anal., 25(1994), 1433-1466. MR 96f:39009

[14] H. Volkmer, On the regularity of wavelets, IEEE Trans. Inform. Theory, 38(1992), 872-876. MR 93f: 42058

[15] H. Volkmer, Asymptotic regularity of compactly supported wavelets, SIAM J. Math. Anal., 26(1995), 1075-1087. MR 96h:42031

(K. S. Lau) Department of Mathematics, University of Pittsburgh, Pittsburgh, PennSYLVANIA 15260

Institute of Mathematical Sciences, The Chinese University of Hong Kong, Shatin, HONG KONG

(Q. Sun) Center for Mathematical Sciences, Zhejiang University, Hangzhou 310027 , CHINA

Current address: Department of Mathematics, National University of Singapore, 10 Kent Ridge Crescent, Singapore

E-mail address: matsunqy@leonis.nus.edu.sg 